\title{
Importancia de los servicios ecosistémicos del recurso vegetal de la parroquia San Juan, cantón Riobamba
}

\author{
Importance of the ecosystem services of the vegetable resource of the \\ parish San Juan, canton Riobamba
}

\author{
Marcela Yolanda Brito Mancero. ${ }^{1}$, Mary Silvana Moreta Escobar. ${ }^{2}$, Alex Vinicio \\ Gavilanes Montoya. ${ }^{3} \&$ Zoila Valeria Tapia González. ${ }^{4}$
}

\begin{abstract}
.
DOI: https://doi.org/10.33262/cienciadigital.v4i3.1333

Knowing the present relationship between the benefits provided by the service of plant ecosystems with the importance and frequency of use assigned by the inhabitants allows establishing an assessment of the resource. Twenty ecosystem services distributed in: $\mathrm{s}$ were identified. regulation (7), s. provisioning (6), s. support (3) and s. cultural (4) by using the methodology of the Inter-American Development Bank (IDB) which is based on the study of economic conditions and activities of the population, climatic conditions, identification of the importance of forest conservation, land and water use, current regulations, and the powers of sectional governments. 326 surveys were applied to the population, the information was processed using non-parametric tests and the inferential analytical method, determining that the population is willing to pay for conservation; variables ethnicity, gender and level of education are those that assign the values of importance and tangible ecosystem services are those that have a higher valuation.
\end{abstract}

Keywords: Rural parish, Riobamba, San Juan, ecosystem services, forest resources.

\footnotetext{
${ }^{1}$ Docente Investigador, Facultad de Ciencias, Escuela Superior Politécnica de Chimborazo, Riobamba, mybrito@espoch.edu.ec

${ }^{2}$ Investigador independiente en el área ambiental, Riobamba, msilvanamoreta@yahoo.es

3 Docente Investigador, Extensión Orellana, Escuela Superior Politécnica de Chimborazo, Puerto Francisco de Orellana, vinicio.gavilanes@espoch.edu.ec

${ }^{4}$ Escuela Superior Politécnica de Chimborazo, Instituto de post grado y educación continua, Riobamba, ztapia@espoch.edu.ec
} 


\section{Resumen.}

El conocer la relación presente entre los beneficios que proporciona el servicio de los ecosistemas vegetales con la importancia y frecuencia de uso que le asignan los pobladores permite establecer una valoración al recurso. Se identificaron 20 servicios ecosistémicos distribuidos en: s. regulación (7), s. aprovisionamiento (6), s. soporte (3) y s. culturales (4) mediante uso de la metodología del Banco Interamericano de Desarrollo (BID) que se basa en el estudio de condiciones y actividades económicas de la población, condiciones climáticas, identificación de la importancia de la conservación de bosques, uso del suelo y agua, normativa vigente, y las competencias de los gobiernos seccionales. Se aplicaron 326 encuestas a la población, el procesamiento de la información se lo realizó mediante pruebas no paramétricas y el método analítico inferencial, determinando que la población está dispuesta a pagar por conservación; las variables etnia, género y nivel de educación son los que asignan los valores de importancia y los servicios ecosistémicos tangibles son los que poseen una valoración más alta.

Palabras clave: Parroquia rural, Riobamba, San Juan, servicios ecosistémicos, recursos forestales.

\section{Introducción.}

Los servicios ambientales se clasifican en: aprovisionamiento, regulación (Haines-Young \& Potschin, 2013); culturales (Rengifo, Rios, Fachín, \& Vargas, 2017), y de soporte (Popa \& Bann, 2012). Los bienes o servicios ecosistémicos que presta la naturaleza son consumidos de forma directa, mediante la interacción entre sí, que mejoran el desarrollo y la calidad de vida de las personas (Balvanera, P., 2012).

Algunos servicios no poseen valores monetarios de mercado y se necesita tiempo para asignar valor para ellos (Izco \& Burneo, 2003); la influencia que tiene las partes interesadas (Lamarque, Quétier, \& Lavorel, 2011); sobre los servicios puede ser positiva o negativa (Felipe, Comín, \& Escalera, 2015). Durante la identificación de los servicios ambientales requiere de un proceso interactivo y de consulta regular, estableciendo preguntas claves y concisas y definir indicadores de uso (Brown, et al., 2014).

Expresando de tal manera una necesidad de estudio para la parroquia San Juan mediante la determinación de la importancia de los servicios, la identificación de índices ecológicos y la dependencia de los mismos por los factores sociales (Lundberg \& Moberg, 2003); relación población y el entorno ambiental (Nottea, et al., 2017).

Mediante la encuesta se construyó en torno a la metodología clave de: servicios derivados únicamente de los ecosistemas locales y que un flujo de servicios (uso real) colocar solo cuando dichos servicios se consumen directamente (disfrutado) por el destinatario final (Corral, 2010).

Además de conocer la importancia de la conservación de bosques y peligros que enfrentan los ecosistemas, motivos del desgaste de los recursos, afectaciones culturales (Milcu, Hanspach, Abson, \& Joern, 2013); conocimiento de las entidades responsables de la protección, conservación del área protegida, importancia y frecuencia de uso de los 
servicios, actividades comerciales y egresos, motivos por los cuales no estarían dispuestos a pagar (Kauyeken, 2014); y la disponibilidad de pago por la conservación de los bosques (Peixer, Giacomini, \& Petrere, 2011).

Conjuntamente con el aporte investigativo permite reconocer la importancia que tienen los servicios para la economía y la sociedad (Spangenberg, Haaren, \& Settele, 2014). Identifican la alta demanda de bienes y servicios vinculado al aumento poblacional (Groota, Wilson, \& Boumansb, 2002), como uno de los factores de la degradación continua del ambiente, a los cuales hay que sumarle los cambios en el uso de suelo (Tsonkovaa, Quinkensteina, Böhma, Freesea, \& Schaller, 2014); el cambio climático (Lamarque, Tappeiner, Turner, \& Steinbacher, 2011); entre otros que influyen al acelerado daño ambiental (Oropeza, Urciaga, \& Ponce, 2015).

El reconocer las prioridades sociales en cuanto a la importancia y frecuencia de uso así como la identificación de los servicios favorecerá la aplicación de acciones para su preservación (Campaña, 2015); y reparación del ambiente a 4 categorías: conservación, restauración pasiva y activa, uso sustentable; donde se pueden implementar medidas para un manejo sostenible por zona (GADP-San Juan, 2015).

El impacto directo que tendrá esta investigación será sobre los 8443 habitantes de la parroquia y el gobierno autónomo descentralizado de la localidad; quienes son los beneficiaros de estos servicios ecosistémicos, mientras que de manera indirecta serán las autoridades provinciales, sectoriales y organizaciones no gubernamentales, como parte de la planificación territorial.

\section{Metodología.}

Identificación y consulta a las partes involucradas "stakeholders" y revisión bibliográfica. Se aplicaron encuestas a los habitantes de la población y entrevistas a los líderes barriales siguiendo la recomendación del Banco Interamericano de Desarrollo (BID) (Herrera, Felipe, 2017). Como fuente secundaria se revisó el inventario vegetal, población zonal, sistema comunitario, culturas, sustentabilidad social y económico en el plan de desarrollo y ordenamiento territorial local, cantonal y provincial (FAO, 2009).

Tabla 1: Identificación de los "stakeholders" de la parroquia

\begin{tabular}{ll}
\hline \multicolumn{1}{c}{ Partes interesadas } & $\begin{array}{l}\text { Partes involucradas que están trabajando en el } \\
\text { manejo de los recursos ambientales }\end{array}$ \\
\hline & $\begin{array}{l}\text { Ministerio de Ambiente del Ecuador } \\
\text { Ministerio de Agricultura y Pesca }\end{array}$ \\
Juntas comunales de pueblos indígenas & Gobierno Autónomo Descentralizado Municipal \\
Propietarios de bosques (propiedad privada) & de Riobamba \\
Organizaciones de productores & Gobierno Autónomo Descentralizado Provincial \\
Gobierno Autónomo Descentralizado Parroquial & de Chimborazo \\
de San Juan & Escuela Superior Politécnica de Chimborazo \\
Sociedad local & Universidad Nacional de Chimborazo \\
& ECOPAR \\
& PROMAREN \\
\hline
\end{tabular}


Fuente: Gobierno Autónomo Descentralizado parroquial San Juan,2018

El cálculo de la muestra se realizó el cálculo de n-encuestas aplicado para poblaciones finitas (Aguilar, 2005), para un universo poblacional de 2126, obteniendo 326 entrevistas a aplicarse, distribuidas de acuerdo con su densidad poblacional.

Tabla 2: Distribución de encuestas por barrios (Cabecera parroquial San Juan)

\begin{tabular}{lccc}
\hline Barrio & $\begin{array}{c}\text { **Promedio de } \\
\text { integrantes (4) }\end{array}$ & Porcentaje & $\mathbf{N}^{\circ}$ encuestas \\
\hline San Francisco & 352 & 16,6 & 54 \\
San Vicente & 313 & 14,7 & 48 \\
Central & 365 & 17,2 & 56 \\
Santa Marianita & 515 & 24,2 & 79 \\
Rumipamba & 581 & 27,3 & 89 \\
TOTAL & 2126 & 100,0 & 326 \\
\hline
\end{tabular}

Fuente: Elaboración propia.

El área de aplicación se obtuvo de los valores de importancia y frecuencia de uso de los servicios, se identificó sitios en los que: los pobladores de la parroquia local son altamente dependientes de la naturaleza ya que con ella obtienen su capital de ingreso y beneficios (Affek \& Kowalska, 2017).

Identificación de los servicios ecosistémicos que proporciona el recurso vegetal se realizó mediante conversatorios centrados hacia los actores principales se identificaron los servicios prestados por la vegetación distribuidos entre las categorías: aprovisionamiento, regulación, soporte y culturales.

Para el desarrollo de la encuesta se elaboraron preguntas básicas detenidamente expuestas a revisión, adaptadas a la realidad de la zona de estudio después de haber recopilado información por parte de las autoridades locales, cantonales provinciales y personas voluntarias con criterio que habitan en la parroquia.

A los encuestados se les indicó que limitaran sus consideraciones a los servicios proporcionados por los ecosistemas locales. Los servicios ecosistémicos identificados se distribuyeron por categorías en los cuales se obtuvo: 6 de aprovisionamiento, 7 de regulación, 3 de soporte y 4 culturales (Affek \& Kowalska, 2017).

Para la evaluación de los servicios ecosistémicos se eligió un rango corto de calificación se aplicó una escala numérica del 1 al 5 ante los servicios enlistados ya sea por importancia o frecuencia de uso. 
Tabla 3. Escala de colores para la evaluación de los servicios identificados según la importancia y la frecuencia de uso

\begin{tabular}{llcccc}
\hline $\mathbf{1}-\mathbf{1 , 9 9}$ & $\mathbf{2 - 2 , 9 9}$ & $\begin{array}{c}\text { VALORES } \\
\mathbf{3} \mathbf{3 , 9 9}\end{array}$ & $\mathbf{4 - 4 , 9 9}$ & $\mathbf{5}$ \\
\hline *I & $\begin{array}{c}\text { Poco o nada } \\
\text { importante }\end{array}$ & $\begin{array}{c}\text { Algo } \\
\text { importante }\end{array}$ & $\begin{array}{c}\text { Medianamente } \\
\text { importante }\end{array}$ & Importante & Muy importante \\
$\begin{array}{c}\text { Poco o nada } \\
\text { frecuente }\end{array}$ & $\begin{array}{c}\text { Algo } \\
\text { frecuente }\end{array}$ & $\begin{array}{c}\text { Medianamente } \\
\text { frecuente }\end{array}$ & Frecuente & Muy frecuente \\
\hline
\end{tabular}

*I= Importancia del servicio ecosistémico, $* \mathrm{~F}=$ Frecuencia de uso del servicio ecosistémico

Fuente: Elaboración propia.

Para averiguar la importancia y la frecuencia del uso otorgado a los servicios provenientes de los ecosistemas se obtuvo el promedio de las respuestas emitidas por los encuestados según la escala de Likert del 1 al 5, diferenciado a la importancia del servicio con la de frecuencia de uso del servicio.

Elementos que cambiaron la percepción del ecosistema se realizó mediante el análisis estadístico desarrollado con los resultados obtenidos de las encuestas aplicadas a la población, se consideró los siguientes factores (variables independientes): barrio, género, etnia y nivel de instrucción.

Tabla 4: Pruebas aplicadas en el análisis estadístico.

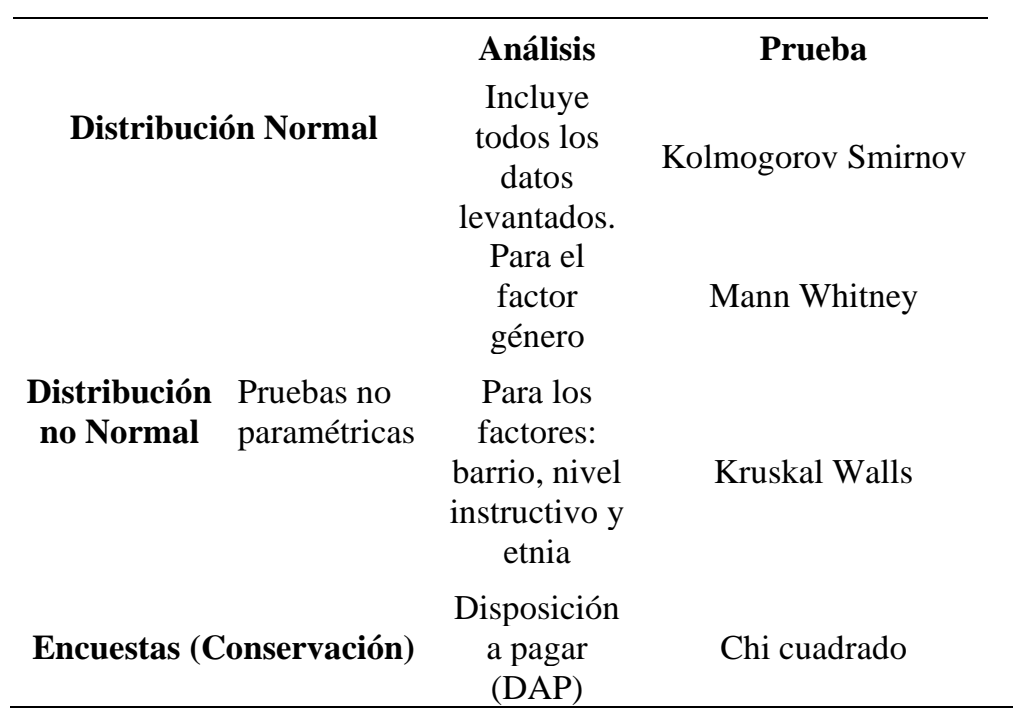

Fuente: Elaboración propia.

\section{Resultados y Discusión.}

Resultados de selección de los servicios mediante las entrevistas realizadas 
Para el proceso de evaluación de los servicios ecosistémicos se modificaron los términos con los que se usó en las entrevistas y encuestas por un lenguaje técnico ambiental para nuestro estudio.

Tabla 5: Resultado del proceso de entrevista hacia los usuarios con nivel de decisión

\begin{tabular}{llc}
\hline \multicolumn{1}{c}{ Categorías } & \multicolumn{1}{c}{ Servicios Ecosistémicos } & $\begin{array}{c}\text { Calificación De Los } \\
\text { Servicios } \\
\text { Seleccionados (\%) }\end{array}$ \\
& Alimentación humana & 100 \\
Servicios & Alimentación animal & 100 \\
aprovisionamiento & Madera para construcción & 60 \\
& Plantas medicinales & 60 \\
& Polinización & 80 \\
& Leña & 45 \\
Servicios & Regulación del ciclo hidrológico & 100 \\
regulación & Incremento de filtración & 80 \\
& Conservación de agua por los bosques & 100 \\
de & Regulación del microclima & 80 \\
& Prevención de deslizamientos de tierra & 80 \\
Servicios de soporte & Prevención de inundaciones & 80 \\
& Control biológico & 80 \\
& Vegetación que ayuda a mantener los & 100 \\
& niveles de agua & 100 \\
& Ciclo de nutrientes en el suelo & 80 \\
Servicios culturales & Residuos orgánicos que mejoran el suelo & 80 \\
& Descanso, relajación & 70 \\
& Recreación & 100 \\
& Paisaje & 50 \\
\hline & Prácticas ancestrales & \\
\hline
\end{tabular}

Fuente: Elaboración propia.

En vista de los altos valores porcentuales registrados durante las entrevistas realizadas se obtuvo que la mayor parte de los servicios ecosistémicos enlistados son de suma preferencia en la parroquia San Juan, donde fueron supervisados y aprobados para realizar las evaluaciones, sin embargo, es notorio que los usuarios reconocen y valoran los beneficios ambientales prestados por la vegetación.

Los servicios que tienen un valor menor como por ejemplo la madera o los productos forestales maderables, se debe a la diferencia de criterio de las personas entrevistadas debido a que una parte lo usan como combustible y para ambientar sus casas con calor por las bajas temperaturas de la zona; pero, en otros casos se considera como un recurso que está en déficit y que no se debería usar.

Caracterización de la encuesta para el proceso de evaluación de los servicios prestados se realizó del total de muestra a encuestar dio 326 distribuidas en los 5 barrios de la cabecera parroquial (Tabla 2) y en base a este número se realizaron los análisis estadísticos, mismos 
que se resumen con los factores de mayor proporción que inciden en el uso de los servicios ecosistémicos y se muestran a continuación:

Tabla 6: Resultados del análisis porcentual de los factores en estudio

\begin{tabular}{c|cc}
\hline & Factores & Distribución porcentual \\
\hline \multirow{3}{*}{ Etnia } & Indígena & $\mathbf{6 2 , 9 0}$ \\
& Mestiza & 36,20 \\
& Blanco & 9,00 \\
\hline \multirow{2}{*}{ Género } & Femenino & 48,80 \\
& Masculino & $\mathbf{5 1 , 2 0}$ \\
\hline \multirow{2}{*}{ Edad } & $30-45$ años & $\mathbf{3 1 , 3 0}$ \\
& $18-29$ años & 29,40 \\
\hline \multirow{3}{*}{ Nivel de } & Primaria & 43,30 \\
instrucción & Secundaria & $\mathbf{3 0 , 1 0}$ \\
& Tercer nivel & 16,60 \\
& Cuarto nivel & 9,20 \\
\hline \multirow{3}{*}{ Ocupación } & Ninguna & $\mathbf{4 5 , 1 0}$ \\
& Agricultura & 21,70 \\
& Otros (quehaceres doméstico, & 10,10 \\
& chofer, guardián) & \\
\hline
\end{tabular}

Fuente: Statistical Package for the Social Sciences.

Importancia y frecuencia de uso de los servicios provistos por los ecosistemas.

Se fijaron 20 servicios ecosistémicos en este estudio para los análisis de forma independiente, en base a su importancia y a su frecuencia, lo que representó un total de 40 repeticiones por análisis para cada servicio.

Tabla 7: Evaluación de los servicios ecosistémicos mediante los valores medios (VM) obtenidos en el análisis estadístico de acuerdo a la IMPORTANCIA (*I) y FRECUENCIA DE USO $(* \mathrm{~F})$ de los mismos

\begin{tabular}{|c|c|c|c|c|c|c|c|c|c|c|c|c|c|c|c|c|c|c|c|c|}
\hline EC. & & & VEGE & TAL & & & $\overline{A C}$ & UÁTI & $\mathbf{O O}$ & & & & & VEC & SETA & & & & & \\
\hline \multirow{2}{*}{ 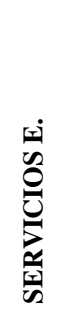 } & 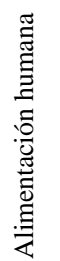 & 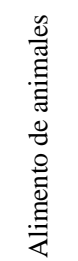 & 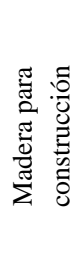 & 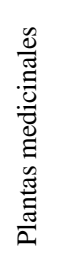 & 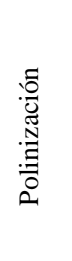 & 苟 & 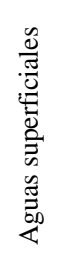 & 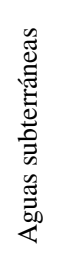 & 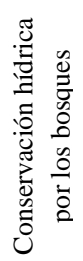 & 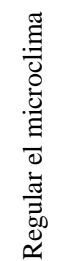 & 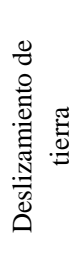 & 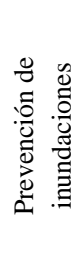 & 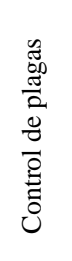 & 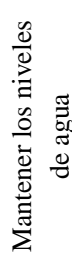 & 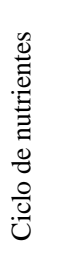 & 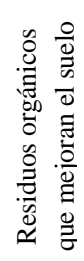 & 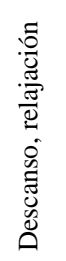 & 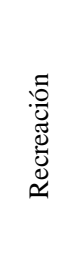 & 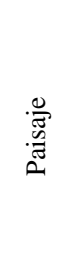 & 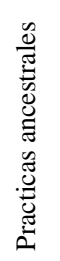 \\
\hline & \multicolumn{6}{|c|}{ APROVISIONAMIENTO } & \multicolumn{7}{|c|}{ REGULACIÓN } & \multicolumn{4}{|c|}{ SOPORTE } & \multicolumn{3}{|c|}{ CULTURAL } \\
\hline$I^{*}$ & 4,79 & 4,40 & 3,32 & 4,34 & 3,81 & 3,87 & 4,09 & 4,06 & 4,42 & 4,08 & 4,19 & 4,22 & 4,07 & 4,41 & 4,45 & 4,50 & 4,51 & 4,20 & 4,64 & 3,32 \\
\hline $\mathbf{F}^{*}$ & 4,56 & 4,10 & 2,69 & 3,90 & 3,14 & 3,40 & 3,51 & 3,52 & 3,98 & 3,75 & $\mathbf{3 , 8 3}$ & 3,81 & 3,61 & 4,02 & 4,12 & 4,10 & 3,96 & 3,47 & 4,48 & 2,75 \\
\hline
\end{tabular}

Fuente: Elaboración propia. 
Se visualiza principalmente que el valor de mayor importancia que los usuarios le dan a los bienes generados por la naturaleza, son los servicios de aprovisionamiento entorno a la alimentación humana.

Los de menor valor son la madera para construcción debido a la escasez en el sector, mientras que las practicas ancestrales relacionadas a los servicios culturales tienen una importancia media, ya que, a pesar de ser una parroquia rural con predominancia del sector indígena, sus tradiciones van siendo modificadas por el mestizaje.

En cuanto a la frecuencia de uso de los servicios, los usuarios otorgaron una alta importancia en lo que respecta a la alimentación humana, esto se debe a que la actividad que más desarrollan en el sector es la agricultura y es el sustento para sus familias. Además, se calificaron con valores altos los servicios de regulación donde está el agua, ya que es el medio que permite el desarrollo de la producción agrícola.

La variación del uso de las prácticas ancestrales es un servicio que aún tiene apogeo, sin embargo, con el pasar de los años estas prácticas religiosas se irán desapareciendo por la asimilación y adaptación de otras costumbres que debilitan el fortalecimiento espiritual (Rengifo, Rios, Fachín, \& Vargas, 2017).

Los servicios de regulación tienen un valor alto, ya que existe una relación cercana entre los recursos (agua-vegetación) y cierta dependencia entre ellos (Orellana \& Espadas, 2008). Aquellos servicios tangibles son los que la población mayoritariamente asigna unas valoraciones más altas (Affek \& Kowalska, 2017). Cabe mencionar además que los beneficios de los servicios ambientales permiten proveer y abastecer las necesidades de los usuarios (Affek \& Kowalska, 2017).

Tabla 8: Dispersión de los datos (desviación estándar) en cuanto a la importancia $(* \mathrm{I})$ y frecuencia $(* \mathrm{~F})$ de uso de los servicios ecosistémicos.

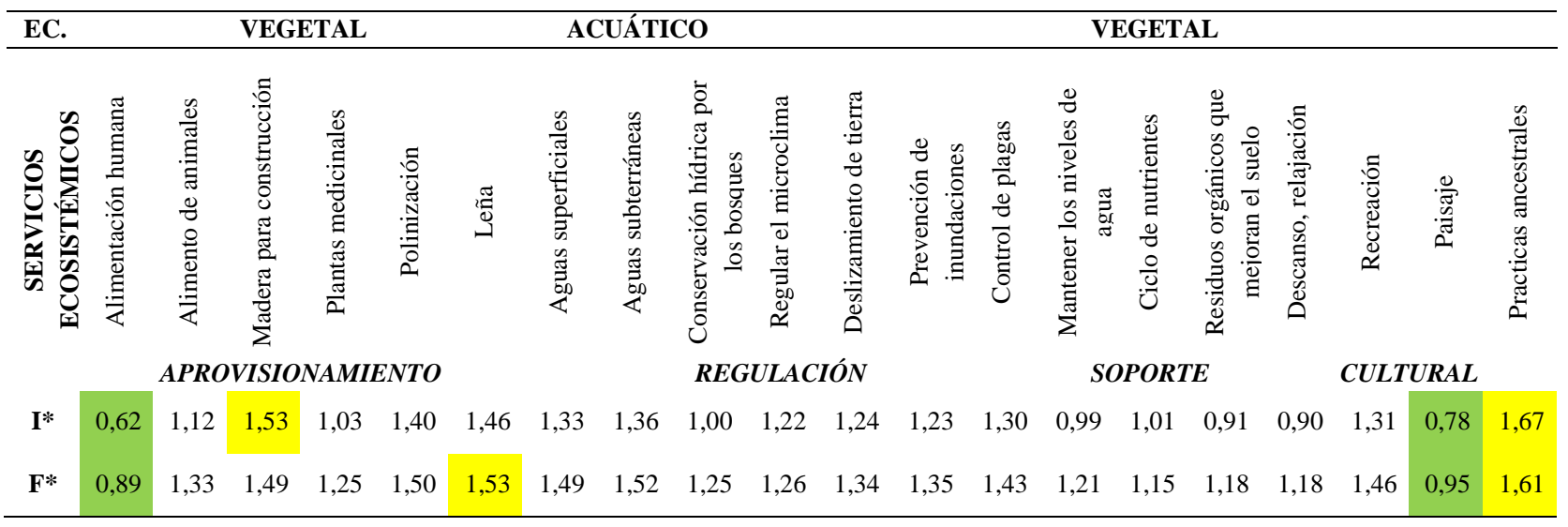

Fuente: Elaboración propia.

Las celadas marcadas en la Tabla 8, muestran los servicios de mayor impacto que tuvieron sobre población de la parroquia. La dispersión de los datos respecto al valor promedio obtenido de la encuesta referida a la importancia de los servicios provistos por los ecosistemas tanto vegetal y acuático, por lo que, existe baja variabilidad en las respuestas de los usuarios para la alimentación humana considerada como servicio de 
aprovisionamiento y al paisaje como servicio cultural. A su vez, el de mayor dispersión es la madera utilizada para construcción y en el caso de los servicios culturales son las prácticas ancestrales.

Para la frecuencia de uso ocurre lo mismo que la importancia con la diferencia que existe variabilidad en las preferencias de uso de madera como combustible (leña). Finalmente, los datos no normales ocurren por el desconocimiento del usuario acerca de los beneficios que prestan los bienes y servicios ambientales.

Resultados del análisis mediante pruebas no paramétricas de los factores que modifican la percepción en cuanto a la importancia (I) y frecuencia (F) de uso de los servicios ecosistémicos.

Se aplicaron pruebas no paramétricas para cada factor en relación a los servicios ecosistémicos, para determinar cuál de ellos depende de las condiciones sociodemográficas (etnia, género, nivel de instrucción y barrio). Si el valor obtenido es menor al 0,05 de significancia, se considera que ese servicio ecosistémico es modificado por el factor.

Tabla 9: Factores que modifican la percepción en cuanto a la importancia (I) y frecuencia $(\mathrm{F})$ de uso de los servicios ecosistémicos.

\begin{tabular}{|c|c|c|c|c|c|c|c|c|c|}
\hline \multirow[b]{2}{*}{ CATEG. } & \multirow[b]{2}{*}{ SERVICIO ECOSISTÉMICO } & \multicolumn{2}{|c|}{ ETNIA } & \multicolumn{2}{|c|}{ GÉNERO } & \multicolumn{2}{|c|}{ N. INSTRUCCIÓN } & \multicolumn{2}{|c|}{ BARRIO } \\
\hline & & $\mathbf{I}$ & $\mathbf{F}$ & I & $\mathbf{F}$ & I & $\mathbf{F}$ & I & $\mathbf{F}$ \\
\hline \multirow{6}{*}{ 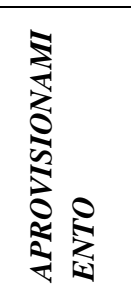 } & Alimentación humana & 0,528 & 0,980 & 0,109 & 0,161 & 0,141 & 0,039 & 0,074 & $\mathbf{0 , 0 3 2}$ \\
\hline & Alimentación animal & 0,162 & $\mathbf{0 , 0 4 7}$ & 0,528 & 0,019 & 0,597 & 0,635 & $\mathbf{0 , 0 0 0}$ & 0,009 \\
\hline & Madera para construcción & 0,354 & 0,668 & 0,329 & 0,411 & 0,180 & 0,203 & $\mathbf{0 , 0 0 0}$ & 0,002 \\
\hline & Plantas medicinales & 0,104 & 0,103 & 0,076 & 0,010 & 0,094 & 0,379 & $\mathbf{0 , 0 0 0}$ & 0,239 \\
\hline & Polinización & 0,063 & 0,402 & 0,438 & 0,548 & 0,205 & 0,554 & $\mathbf{0 , 0 3 2}$ & $\mathbf{0 , 0 0 7}$ \\
\hline & Leña & $\mathbf{0 , 0 0 0}$ & $\mathbf{0 , 0 0 0}$ & 0,007 & 0,007 & 0,000 & $\mathbf{0 , 0 0 0}$ & 0,009 & $\mathbf{0 , 0 0 8}$ \\
\hline \multirow{7}{*}{ 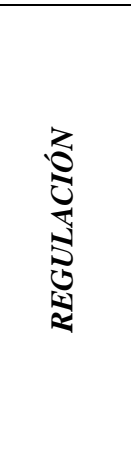 } & $\begin{array}{l}\text { Regulación del ciclo } \\
\text { hidrológico }\end{array}$ & 0,494 & 0,564 & 0,431 & 0,924 & 0,086 & 0,002 & $\mathbf{0 , 0 0 0}$ & 0,207 \\
\hline & Incremento de filtración & 0,347 & 0,804 & 0,890 & 0,876 & 0,151 & 0,019 & $\mathbf{0 , 0 0 0}$ & 0,120 \\
\hline & $\begin{array}{l}\text { Conservación de agua por los } \\
\text { bosques }\end{array}$ & 0,244 & $\mathbf{0 , 0 3 5}$ & 0,364 & 0,686 & 0,051 & 0,016 & $\mathbf{0 , 0 0 0}$ & $\mathbf{0 , 0 0 2}$ \\
\hline & Regulación del microclima & 0,483 & 0,249 & 0,631 & 0,921 & 0,073 & 0,022 & $\mathbf{0 , 0 0 0}$ & $\mathbf{0 , 0 0 0}$ \\
\hline & $\begin{array}{l}\text { Prevención de deslizamientos } \\
\text { de tierra }\end{array}$ & 0,018 & $\mathbf{0 , 0 0 3}$ & 0,465 & 0,786 & 0,130 & 0,181 & $\mathbf{0 , 0 0 3}$ & 0,136 \\
\hline & Prevención de inundaciones & 0,488 & 0,133 & 0,054 & 0,155 & 0,369 & 0,247 & $\mathbf{0 , 0 0 0}$ & $\mathbf{0 , 0 0 8}$ \\
\hline & Control biológico de plagas & 0,452 & 0,758 & 0,213 & 0,975 & 0,127 & 0,055 & $\mathbf{0 , 0 0 0}$ & $\mathbf{0 , 0 2 6}$ \\
\hline \multirow{3}{*}{ 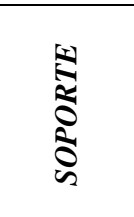 } & $\begin{array}{l}\text { Vegetación que ayuda a } \\
\text { mantener los niveles de agua }\end{array}$ & 0,137 & 0,512 & 0,587 & 0,604 & 0,251 & $\mathbf{0 , 0 0 1}$ & 0,004 & $\mathbf{0 , 0 1 4}$ \\
\hline & Ciclo de nutrientes en el suelo & 0,995 & 0,702 & 0,003 & 0,081 & 0,326 & 0,350 & 0,000 & 0,024 \\
\hline & $\begin{array}{l}\text { Residuos orgánicos que } \\
\text { mejoran el suelo }\end{array}$ & 0,484 & 0,588 & 0,699 & 0,501 & 0,245 & 0,372 & 0,001 & 0,571 \\
\hline \multirow{4}{*}{ 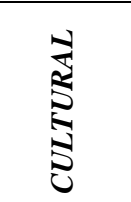 } & Descanso, relajación & 0,285 & 0,732 & 0,649 & 0,983 & 0,592 & 0,730 & 0,029 & 0,285 \\
\hline & Recreación & 0,091 & 0,428 & 0,002 & 0,000 & $\mathbf{0 , 0 2 3}$ & 0,002 & 0,001 & $\mathbf{0 , 0 0 0}$ \\
\hline & Paisaje & 0,128 & 0,083 & 0,436 & 0,080 & 0,309 & 0,166 & $\mathbf{0 , 0 0 0}$ & $\mathbf{0 , 0 0 0}$ \\
\hline & Prácticas ancestrales & 0,002 & 0,031 & 0,307 & 0,252 & 0,001 & 0,000 & $\mathbf{0 , 0 0 0}$ & 0,010 \\
\hline
\end{tabular}

Fuente: Elaboración propia. 
Los factores de mayor incidencia que modifican la percepción en torno a la importancia de los servicios ecosistémicos y su frecuencia de uso fueron: etnia, género y nivel de instrucción.

La edad cambia la percepción de la importancia de los servicios provistos (Affek \& Kowalska, 2017), va además, en base a la formación educativa y al conocimiento ambiental (Allendorf \& Yang, 2013); este último factor no fue considerado en este estudio.

Las variaciones que provienen de los diferentes factores analizados mediante el valor medio de frecuencia e importancia se resumen en la siguiente tabla:

Tabla 10: Resultados del análisis del valor medio (VM) de la importancia y frecuencia de uso de los servicios ecosistémicos en función de los factores sociales y el factor geográfico.

\begin{tabular}{|c|c|c|c|}
\hline \multicolumn{2}{|c|}{ FACTORES } & IMPORTANCIA & FRECUENCIA \\
\hline \multirow{3}{*}{ Etnia } & Indígena & Alimentación humana & Alimentación humana \\
\hline & Mestiza & Alimentación humana & Paisaje \\
\hline & Blanco & Plantas medicinales & Plantas medicinales \\
\hline \multirow[b]{2}{*}{ Sexo } & Femenino & Alimentación humana & Alimentación humana \\
\hline & Masculino & Alimentación humana & Alimentación humana \\
\hline \multirow{13}{*}{$\begin{array}{l}\text { Nivel de } \\
\text { instrucción }\end{array}$} & Ninguna & Alimentación humana & Alimentación humana \\
\hline & Primaria & Alimentación humana & Alimentación humana \\
\hline & Secundaria & Alimentación humana & Paisaje \\
\hline & Tercer nivel & Alimentación humana & Alimentación humana \\
\hline & \multirow{9}{*}{ Cuarto nivel } & Aguas subterráneas & \multirow{9}{*}{ Deslizamiento de tierra } \\
\hline & & Regular el microclima & \\
\hline & & Deslizamiento de tierra & \\
\hline & & Prevención de & \\
\hline & & $\begin{array}{l}\text { inundaciones } \\
\text { Control biológico }\end{array}$ & \\
\hline & & Mantener los niveles del & \\
\hline & & agua & \\
\hline & & Descanso & \\
\hline & & Paisaje & \\
\hline \multirow{5}{*}{ Barrio } & San Francisco & Alimentación humana & Alimentación humana \\
\hline & San Vicente & Alimentación humana & Paisaje \\
\hline & Central & Alimentación humana & Alimentación humana \\
\hline & Santa Marianita & Alimentación humana & Alimentación humana \\
\hline & Rumipamba & Paisaje & Paisaje \\
\hline
\end{tabular}

Fuente: Elaboración propia.

Como se observa en la Tabla 10, los servicios de mayor importancia se destacan la alimentación humana para los factores analizados, la etnia blanca reconoce la importancia de las plantas medicinales, y la población mestiza el uso frecuente del paisaje como servicio cultural. 
La etnia es un factor que incide en las preferencias de servicios ambientales, por lo tanto, se debe considerar esta variable para establecer planes de desarrollo (Cuni, Pfeifer, Marchant, \& Burgess, 2016). Asimismo, las prioridades de conservación pueden ser establecidas según las tendencias sociodemográficas (Graves, Pearson, \& Turnera, 2017). La variable género no presenta diferencias en lo referente a labores tanto hombres como mujeres realizan actividades semejantes. Asimismo, para esta variable género se estable que el servicio ecosistémico alimentación humana en importancia y frecuencia de uso se considerado como un regulador entre el ambiente y la potencialidad del recursos (Garcia, Spence, \& McGannon, 2005).

Basados en estudios realizados por (Affek \& Kowalska, 2017) que indica que en determinados casos los roles realizados por el género masculino se orientan a actividades de manejo de los bosques y cultivos mientras que a la mujer se encarga el cuidado del hogar y quehaceres doméstico; se puede inferir que el análisis de este factor es variable ya que en estudios subsiguientes se observa la equidad del género que se da en algunos sectores y por ende las labores igualitarias.

La mayor cantidad de servicios favorecidos con puntuaciones altas son realizados por personas con título de cuarto nivel (Allendorf \& Yang, 2013). Se debe al conocimiento en temas de conservación y desarrollo que dicha población posee. El valor medio de los servicios varía en función del factor barrio, esto es en consecuencia de que la percepción depende del pensamiento social que no es similar de un barrio a otro por la distinción de criterios, necesidades y accesibilidad.

Resultados del análisis estadístico de las preguntas vinculadas al DAP.

Tabla 11: Dependencia de la DAP con relación a las variables utilizadas

\begin{tabular}{ccc}
\hline Variables independientes & Significancia & Dependencia \\
\hline Género & 0,176 & NO \\
Etnia & 0,159 & NO \\
Edad & 0,125 & NO \\
Estado civil & 0,494 & NO \\
Nivel de instrucción & $\mathbf{0 , 0 0 3}$ & SI \\
Conservación bosque & 0,874 & NO \\
Desaparición sino se cuida & 0,828 & NO \\
Ingresos económicos & $\mathbf{0 , 0 0 4}$ & SI \\
\hline
\end{tabular}

Fuente: Elaboración propia.

La variable dependiente disposición a pagar (DAP) se encuentra relacionada a las variables (socio económicas) nivel de instrucción e ingresos económicos según los establece el análisis inferencial (Moreta, 2019). Dicha dependencia se debe a la relación existente entre el índice económico y la preparación académica que éstos tengan (Peixer, Giacomini, \& Petrere, 2011); el vínculo personal denominado "estado civil” también puede afectar esta variable dependiente (González \& Valdivia, 2009). 


\section{Conclusiones:}

- La parroquia rural de San Juan estableció para el recurso vegetación siete (7) servicios de regulación que son: regulación del ciclo hidrológico, incremento en la filtración, conservación del agua por los bosques, regulación del microclima, prevención de los deslizamientos de tierra, prevención de inundaciones y control biológico; seis (6) servicios de aprovisionamiento: alimentación humana, alimentación animal, madera para construcción, plantas medicinales, polinización y leña; cuatro (4) servicios culturales: descanso, recreación, paisaje y prácticas ancestrales y tres (3) servicios de soporte: mantener los niveles de agua, ciclo de nutrientes en el suelo, mejora del suelo; siendo los servicios ambientales más valorados tanto en importancia como en frecuencia de uso los aquellos servicios tangibles Los factores sociodemográficos de con incidencia favorable en la valoración fueron tres (3) etnia, género y nivel de instrucción.

- Los factores nivel de instrucción y los ingresos económicos en los pobladores de la parroquia rural incidieron en la variable disposición a pagar definiendo un excedente de pago por conservación de los bosques. Para este caso particular la disposición a mantener el recurso vegetal con un manejo técnico en coordinación con las autoridades competentes, para un aprovechamiento sostenible se debe a que los participantes del estudio poseen predios forestales.

\section{Referencias bibliográficas:}

Affek, A., \& Kowalska, A. (7 de Julio de 2017). Ecosystem potentials to provide services in the view of direct users. Ecosystem services, 26(1), 184-185,188, 192, 193. doi:https://doi.org/10.1016/j.ecoser.2017.06.017

Aguilar, S. (enero-agosto de 2005). Fórmulas para el cálculo de la muestra en investigaciones de salud. Salud en Tabaco, 11(1-2), 336. Recuperado el Agosto de 2018, de http://www.redalyc.org/pdf/487/48711206.pdf

Aldana, S., Vereda, F., Hidalgo-Alvarez, R., \& de Vicente, J. (2016). Facile synthesis of magnetic agarose microfibers by directed selfassembly. Polymer, 93, 61-64.

Allendorf, T., \& Yang, J. (2013). The role of ecosystem services in park-people relationships: The case of Gaoligongshan Nature Reserve in southwest China (Primera ed., Vol. 167). Biological Conservation. doi:10.1016/j.biocon.2013.08.013

Balvanera, P. (25 de Febrero de 2012). Los servicios ecosistémicos que ofrecen los bosques tropicales. Revista cientîfica y tècnica de medio ambiente ECOSISTEMAS, XXI(1-2), 137-138. Recuperado el 30 de Julio de 2018, de http://www.redalyc.org/pdf/540/54026849011.pdf

Bhat, S., Tripathi, A., \& Kumar, A. (2010). Supermacroprous chitosan-agarose-gelatin cryogels. in vitro characterization and in vivo assesment for cartilage tissue engineering. Journal of the Royal Society Interface, 1-15. 
Bossis, G., Marins, J., Kuzhir, P., Volkova, O., \& Zubarev, A. (2015). Functionalized microfibers for field-responsive materials and biological applications. Journal of Intelligent Material Systems and Structures, 1-9.

Brown, C., Reyers, B., Ingwall-King, L., Mapendembe, A., Nel, J., O'Farrell, P., . . . Bowles-Newark, N. (2014). Measuring ecosystem services: Guidance on developing ecosystem service indicators. (Primera ed.). (K. Trumper, Ed.) Cambridge: United Nations Environment Programme. Recuperado el 2 de Agosto de 2018, de https://www.unepwcmc.org/system/dataset_file_fields/files/000/000/303/original/1850_ESI_Guid ance_A4_WEB.pdf?1424707843

Campaña, F. (2015). La Importancia y el Rol de la Valoración Económica de los Servicios Ambientales para las Negociaciones de Cambio Cl Valoración Económica de Servicios Ambientales de Fijación de C en los Bosques de San Francisco de Borja, Napo-Ecuador. Tesis, 8. Quito: Universidad Andina Simón Bolívar. Obtenido de http://repositorio.uasb.edu.ec/bitstream/10644/4734/1/T1752-MRICampa\%C3\%B1a-La\%20importancia.pdf

Corral, Y. (Julio-Diciembre de 2010). Diseño de cuestionarios para la recolección de datos. Revista Ciencias de la Educación, 20(36), 156-161. Recuperado el 8 de Agosto de 2018, de http://servicio.bc.uc.edu.ve/educacion/revista/n36/art08.pdf

Cortés, J., Puig, J., Morales , J., \& Mendizábal, E. (2011). Hidrogeles nanoestructurados termosensibles sintetizados mediante polimerización en microemulsión inversa. Revista Mexicana de Ingeniería Química., 10(3), 513-520.

Cuni, A., Pfeifer, M., Marchant, R., \& Burgess, N. (2016). Ethnic and locational differences in ecosystem service values: Insights from the communities in forest islands in the desert. Ecosystem Services, 19(1), 43-48. doi:10.1016/j.ecoser.2016.04.004

Dias, A., Hussain, A., Marcos, A., \& Roque, A. (2011). A biotechnological perspective on the application of iron oxide magnetic colloids modified with polysaccharides. Biotechnology Advances 29 , 29, 142-155.

Estrada Guerrero, R., Lemus Torres, D., Mendoza Anaya, D., \& Rodriguez Lugo, V. (2010). Hidrogeles poliméricos potencialmente aplicables en Agricultura. Revista Iberoamericana de Polímeros, 12(2), 76-87.

FAO. (2009). Monitoreo y Evaluación de los Recursos Forestales Nacionales- Manual para la recolección integrada de datos de campo (Tercera ed.). Roma: NFMA 37/S. Recuperado el 8 de Agosto de 2018, de http://www.fao.org/docrep/016/ap152s/ap152s.pdf

Felipe, M., Comín, F., \& Escalera, J. (Mayo de 2015). A framework for the social valuation of ecosystem services. Ambio, XLIV(4), 308-318. doi:10.1007/s 13280014-0555-2 
GADP-San Juan. (2015). Actualización Plan de Desarrollo y Ordenamiento Territorial de la parroquia San Juan (primera ed.). (G.-S. JUAN, Ed.) Riobamba, Chimborazo, Ecuador: "Centro de Estudios Para la Gestión Pública" CEGESPU. Recuperado el 25 de Septiembre de 2018, de http://app.sni.gob.ec/snilink/sni/PORTAL_SNI/data_sigad_plus/sigadplusdocumentofinal/06608235000 01_ACTUALIZACI\%C3\%92N\%20PDYOT\%20SAN\%20JUAN\%2020152019_30-10-2015_22-28-53.pdf

Garcia, E., Spence, J., \& McGannon, K. (13 de Septiembre de 2005). Gender differences in perceived environmental correlates of physical activity. International Journal of Behavioral Nutrition and Physical Activity, II(12), 8-9. doi:10.1186/1479-5868-2-12

García-Cerda, L., Rodríguez-Fernández, O., Betancourt-Galindo, R., Saldívar-Guerrero, R., \& Torres-Torres, M. (2003). Síntesis y propiedades de ferrofluidos de magnetita. Superficies y Vacío., 16(1), 28-31.

González, P., \& Valdivia, R. (2009). Valoración económica de servicios ambientales percibidos en Guanajuato y Silao. Revista Mexicana de Economía Agrícola y de los Recursos Naturales, II(3), 163-177.

Graves, R., Pearson, S., \& Turnera, M. (4 de Abril de 2017). Species richness alone does not predict cultural ecosystem service value. Species richness alone does not predict cultural ecosystem service value, 114(14), 3774-3779. doi:10.1073/pnas.1701370114

Groota, R., Wilson, M., \& Boumansb, R. (2002). A typology for the classification, description and valuation of ecosystem functions, goods and services. Ecological Economics, 41(3), 393-408. doi:https://doi.org/10.1016/S09218009(02)00089-7

Haines-Young, R., \& Potschin, M. (2013). Report to the European Environment Agency. Common International Classification of Ecosystem services. Nottingham: Centre for Environmental Management, University of Nottingham, UK. Recuperado el 31 de Julio de 2018, de https://cices.eu/content/uploads/sites/8/2012/07/CICES-V43_RevisedFinal_Report_29012013.pdf

Herrera, Felipe. (2017). Consulta Significativa con las Partes Interesadas: Series del BID sobre riesgo y oportunidad ambiental y (Primera ed.). Washington: Banco Interamericano de Desarrollo. Recuperado el 30 de Agosto de 2018, de https://publications.iadb.org/bitstream/handle/11319/8454/Consultasignificativa-con-las-partes-interesadas.PDF? sequence $=4 \&$ isAllowed=y

Ilg, P. (2013). Stimuli-responsive hydrogels cross-linked by magnetic nanoparticles. Soft Matter, 9, 3465-3468.

Izco, X., \& Burneo, D. (2003). Herramientas para la valoración y manejo forestal sostenible de bosques sudamericanos. En M. Andelman, \& J. García (Edits.), Unión Mundial para la Naturaleza (Primera ed., págs. 3,15,17,19, 20, 24). Quito, Pinchincha, Ecuador: Publicado por UICN, Oficina Regional para 
América del Sur. Recuperado el 29 de Julio de 2018, de

http://cebem.org/cmsfiles/publicaciones/herramientasdevaloracion.pdf

Kauyeken. (2014). CONOCIMIENTO SOBRE BIODIVERSIDAD: ANÁLISIS

EXPLORATORIO. Santiago de Chile: Creación de un Sistema Nacional Integral de Áreas Protegidas para Chile: Estructura Financiera. Recuperado el 4 de Agosto de 2018, de http://bdrnap.mma.gob.cl/recursos/privados/Recursos/CNAP/GEFSNAP/Kauyeken_2013.pdf

Lamarque, P., Quétier, F., \& Lavorel, S. (2011). Implications de la diversité des définitions du concept de service des écosystèmes pour leur quantification et pour son application à la gestion. Comptes Rendus Biologies, 334(5-6), 441-449. doi:https://doi.org/10.1016/j.crvi.2010.11.007

Lamarque, P., Tappeiner, U., Turner, C., \& Steinbacher, M. (2011). Stakeholder perceptions of grassland ecosystem services in relation to knowledge on soil fertility and biodiversity. Regional Environmental Change, 11(4), 791-804. doi:10.1007/s10113-011-0214-0

Lewitus, D., Branch, J., Smith, K., Callegari, G., Kohn, J., \& Neimark, A. (2011). Biohybrid carbon nanotube/agarose fibers for neural tissue engineering. Advanced Functional Materials, 21, 2624-2632.

Lin, Y.-S., Huang, K.-S., Yang, C.-H., Wang, C.-Y., Yang, Y.-S., Hsu, H.-C., . . T Tsai, C.-W. (2012). Microfluidic synthesis of microfibers for magnetic-responsive controlled drug release and cell culture. PLOS ONE, 7(3), 1-8.

Lundberg, J., \& Moberg, F. (2003). Mobile Link Organisms and Ecosystem Functioning: Implications for Ecosystem Resilience and Management. Ecosystems, 6(1), 0087-0098. doi:https://doi.org/10.1007/s10021-002-0150-4

Martínez, R., Viguera, B., Donatti, C., Harvey, C., \& Alpízar, F. (2017). La importancia de los servicios ecosistémicos para la agricultura (3 ed.). Turrialba: Costa Rica. Obtenido de https://www.conservation.org/publications/Documents/cascade_Modulo-3-Laimportancia-de-los-servicios-ecosistemicos-para-la-agricultura.pdf

Milcu, A., Hanspach, J., Abson, D., \& Joern, F. (2013). Cultural Ecosystem Services: A Literature Review and Prospects for Future Research. Ecology and Society, 18(3), 5-7. Obtenido de https://www.ecologyandsociety.org/vol18/iss3/art44/\#ms_abstract

Moreta, M. (2019). Servicios Ecosistemicos del recurso vegetal en la parroquia San Juan. SECTEI.

Nottea, A., Amato, D. M., Paracchini, M., Liquetea, C., Egohd, B., \& Genelettif, D. (2017). Ecosystem services classification: A systems ecology perspective of the cascade framework. Ecological Indicators, 74, 392-402. doi:http://dx.doi.org/10.1016/j.ecolind.2016.11.030 
Orellana, R., \& Espadas, C. (2008). El papel de la cubierta vegetal en la conservación del agua en la península de Yucatán. En J. Zabala, \& U. a. Yucatán (Ed.), El manejo del agua a través del tiempo en la península de Yucatán (Primera ed., págs. 161-169). Tizimín, Mexico: SEDUMA. Recuperado el 4 de Septiembre de 2018, de http://www.seduma.yucatan.gob.mx/libro-agua-py/capitulo6.pdf

Oropeza, M., Urciaga, J., \& Ponce, G. (2015). Importancia Económica Y Social De Los Servicios De Los Ecosistemas: Una Revisión De La Agenda De Investigación (Economic and Social Importance of Ecosystem Services: A Review of Research Agenda). Revista Global de Negocios, 3(2), 103, 105-108. Recuperado el 2018 de Julio de 2018, de https://papers.ssrn.com/sol3/papers.cfm?abstract_id=2657766

Peixer, J., Giacomini, H., \& Petrere, M. (March de 30 de 2011). Economic valuation of the Emas waterfall, Mogi-Guaçu River, SP, Brazil. Biological Sciences, 83(4), 1287-1301. Recuperado el 8 de Septiembre de 2018, de http://www.scielo.br/pdf/aabc/v83n4/15.pdf

Popa, B., \& Bann, C. (2012). An Assessment of the Contribution of Ecosystems in Protected Areas to Sector Growth and Human Well Being in Romania : Improving the Financial Sustainability of the Carpathian System of Protected Areas (PAs) (Primera ed.). Rumania: Rumania. Recuperado el 1 de Agosto de 2018

Rengifo, E., Rios, S., Fachín, L., \& Vargas, G. (2017). Saberes ancestrales sobre el uso de flora y fauna en la comunidad indígena Tikuna de Cushillo Cocha, zona fronteriza Perú-Colombia-Brasil. Revista peruana de biología, XXIV(1), 77. doi: http://dx.doi.org/10.15381/rpb.v24i1.13108

Ruiz Estrada, G. (2004). Desarrollo de un Sistema de liberación de fármacos basado en nanopartículas magnéticas recubiertas con Polietilénglicol para el tratamiento de diferentes enfermedades. Madrid: Universidad Autónoma de Madrid. Departamento de Física Aplicada.

Song, J., King, S., Yoon, S., Cho, D., \& Jeong, Y. (2014). Enhanced spinnability of narbon nanotube fibers by surfactant addition. Fiberes and Polymers, 15(4), $762-766$.

Spangenberg, J., Haaren, C., \& Settele, J. (2014). The ecosystem service cascade: Further developing the metaphor. Integrating societal processes to accommodate social processes and planning, and the case of bioenergy. Ecological Economics, 104(1), 22-32. doi:https://doi.org/10.1016/j.ecolecon.2014.04.025

Tartaj, P., Morales, M., González-Carreño, T., Veintemillas-Verdaguer, S., \& Serna, C. (2005). Advances in magnetic nanoparticles for biotechnology applications. Journal of Magnetism and Magnetic Materials, 290, 28-34.

Tsonkovaa, P., Quinkensteina, A., Böhma, C., Freesea, D., \& Schaller, E. (2014). Ecosystem services assessment tool for agroforestry (ESAT-A): An approach to assess selected ecosystem services provided by alley cropping systems. Ecological Indicators, 45(1), 285. 
Wulff-Pérez, M., Martín-Rodriguez, A., Gálvez-Ruiz, M., \& de Vicente, J. ( 2013 ).

The effect of polymer surfactant on the rheological properties of nanoemulsions. Colloid and Polymer Science, 291, 709-716.

Zamora Mora, V., Soares, P., Echeverria, C., Hernández, R., \& Mijangos, C. (2015). Composite chitosan/Agarose ferrogels for potential applications in magnetic hyperethermia. Gels., 1, 69-80. 
PARA CITAR EL ARTÍCULO INDEXADO.

Brito Mancero, M. Y., Moreta Escobar, M. S., Alex Vinicio, A. V., \& Tapia González, Z. V. (2020). Importancia de los servicios ecosistémicos del recurso vegetal de la parroquia San Juan, cantón Riobamba. Ciencia Digital, 4(3), 253-270. https://doi.org/10.33262/cienciadigital.v4i3.1333

\section{Ciencia \\ LDigital}

El artículo que se publica es de exclusiva responsabilidad de los autores y no necesariamente reflejan el pensamiento de la Revista Ciencia Digital.

El artículo queda en propiedad de la revista y, por tanto, su publicación parcial y/o total en otro medio tiene que ser autorizado por el director de la Revista Ciencia Digital.
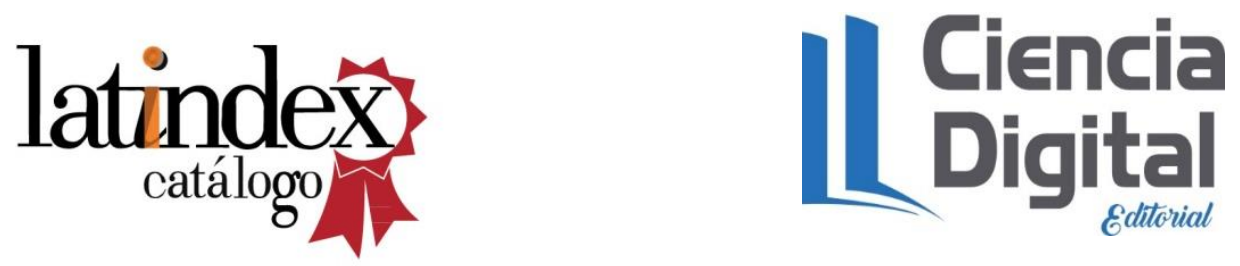